\title{
The impact of hazard-consistent ground motion scenarios selection on structural seismic risk estimation
}

\author{
Mohsen Zaker Esteghamati; ${ }^{1}$ Mahdi Bahrampouri; ${ }^{2}$ and \\ Adrian Rodriguez-Marek, Ph.D. ${ }^{2}$
}

\begin{abstract}
${ }^{1}$ Graduate Research Assistant, Department of Civil and Environmental Engineering, Virginia Polytechnic and State University, Blacksburg, VA, 24060; Email: mohsenzaker@vt.edu

${ }^{2}$ Graduate Research Assistant, Department of Civil and Environmental Engineering, Virginia Polytechnic and State University, Blacksburg, VA, 24060; Email: mahdibp@vt.edu

${ }^{3}$ Professor, Department of Civil and Environmental Engineering, Virginia Polytechnic and State University, Blacksburg, VA, 24060; Email: adrianrm@ vt.edu
\end{abstract}

\begin{abstract}
Structural risk-based evaluation requires a large number of time-history analyses at different ground motion (GM) intensity levels, where the scenarios (e.g. magnitude and distance) of the GMs used in the time-history analyses should be consistent with the site's hazard. The current practice of GM selection typically simplifies the choice of scenario to either an average scenario or the modal scenarios based on the site's hazard deaggregation results. This paper investigates the impact of hazard deaggregation and scenario selection on estimating structural seismic risk. For a hypothetical site in the Eastern US, a Monte Carlo seismic hazard analysis is performed to derive a site-consistent GM suite that captures 1,000,000 years of the site's seismic activity. The complete GM suite consisting of 99,917 records is then used to perform nonlinear dynamic analyses on a mid-rise concrete office building to derive a benchmark seismic demand curve. Subsequently, four GM sets are selected based on average and modal scenarios from two different hazard deaggregation formulation, and the resulting demand curves are compared to the benchmark. The results show that the hazard deaggregation method and scenario choice impacts the demand curve estimation. When deaggregation is performed on IM exceedance, GMs that were selected based on both methods agree well with the benchmark up to higher damage states where mode-based records outperform average-based records. On the other hand, when deaggregation is formulated based on IM occurrence, average scenario-based GMs better match the benchmark, except for higher damage states where again modal scenario-based GMs are in better agreement with the benchmark.
\end{abstract}

\section{INTRODUCTION}

The objective of ground motion (GM) selection methods is to obtain a set of GMs that are consistent with the hazard at a site. Typically, the hazard is determined for a selected intensity measure (IM), which in structural risk estimation applications is commonly taken as pseudo- 
acceleration response spectra at a given period (Zaker Esteghamati et al. 2020). Almost all of the hazard-consistent GM selection methods construct conditional distributions for a full range of spectral periods, conditioned on the hazard for spectral acceleration at a selected period (typically the first mode period of the structure). The selected sets of GM must be consistent with the earthquake scenario(s), in terms of magnitude $(M)$ and distance $(R)$ that contribute to hazard. The difference in these methods originates from the way they establish the target spectra either as uniform hazard spectrum (UHS), conditional spectrum (CS) (Baker 2011; Lin et al. 2013b), or general conditional intensity measure (GCIM) (Bradley 2012; Tarbali and Bradley 2015). In the last decade, a significant body of research has addressed constructing appropriate target spectra and selecting/scaling GMs to match this target (Kohrangi et al. 2020; Kwong and Chopra 2016, 2020; Simon Kwong and Chopra 2017), however, limited studies investigated the impact of hazard scenarios on the structural risk estimation.

To estimate IM spectra, $M$ and $R$ scenarios are commonly chosen based on the site's hazard deaggregation, which relates the exceedance of an IM value to the most likely earthquake events. Although a more precise method is to sample the full deaggregation results and construct IM spectrum for each pair, often a sample statistics such as average is used to construct IM spectrum. While such approximation might hold reasonably accurate for sites with a dominating earthquake scenario, issues arise for sites with multiple contributing earthquake scenarios (Iervolino et al. 2011). Lin et al. (2013) have compared developing CS spectra using only the average hazard scenario from a single ground motion prediction model (GMPM) to one obtained from a full M and R deaggregation from different GMPMs (termed exact CS). They concluded that while two methods are in close agreement, the exact CS method has larger variation at periods far from the conditioning period. They noted that while this difference might lead to increased dispersion in structural response for sites with unimodal deaggregation, it is expected to have a substantial impact on sites with multi-modal deaggregation (Lin et al. 2013a).

This paper investigates the impact of the choice of $\mathrm{M}$ and $\mathrm{R}$ scenario and hazard deaggregation formulation on the structural response estimation as part of a hazard-consistent GM selection method. To this end, a large suite of site-specific GMs is simulated for different earthquake sources of a hypothetical site with similar seismicity to Charleston, South Carolina, following a Monte Carlo seismic hazard analysis. This suite is then applied as the input to structural analyses to assess the seismic performance of a mid-rise concrete office building. The results of the structural analyses are used to construct a "benchmark" demand curve, where the term "benchmark" reflects the fact that the demand curves is build using GMs that cover all possible future earthquakes at the site. Two different hazard deaggregation formulations (occurrence and exceedance) are then performed at eight discrete hazard levels, and two GM sets are selected to match the average and the mode at each hazard level of the two formulations, respectively. Finally, the structural demand curves obtained from the four different choices $(2$ scenarios $\times 2$ hazard deaggregation formulations) are compared to the benchmark demand curve. 


\section{MONTE CARLO SEISMIC HAZARD ANALYSIS}

Site-specific GMs are simulated using Monte Carlo seismic hazard analysis, which estimates the exceedance rate of ground motion IM levels based on the characteristics of all the sources near the site, the GMPM median, and the GMPM standard deviation (Musson 2000). While Monte Carlo seismic analysis has the same inputs and outputs as conventional probabilistic seismic hazard analysis, when used in conjunction with a ground motions simulation model it can generate a suite of ground motions that replicates the distribution of future ground motions at a site. Therefore, this suite of ground motions is used to create a benchmark for the structure's response to future earthquakes. Note that since structural simulations are conducted for the entire suite of future ground motions, estimates of seismic demand do not rely on a ground motion selection approach, justifying the term "benchmark".

Following the seismic source characterization conducted by Chapman and Talwani (2006), two seismic sources at the virtual site are considered. The schematic sources are presented in Figure 1. The first seismic source is a circular zone that produces earthquakes with magnitudes 5 to 7 at the rate of 0.095 per year. The magnitude distribution of events produced by the circular zone follows the truncated Gutenberg-Richter model with a $b$ value equal to 0.87 . The second source is a fault that produces earthquakes with magnitude 7 to 7.5 with a return period of 200 years. The magnitude of earthquakes produced by this fault is uniformly distributed between 7 and 7.5 .

The seismicity of the two sources presented in Figure 1 is simulated for a time window of $1,000,000$ years resulting in an earthquake catalog with 99,917 events. For every earthquake in the

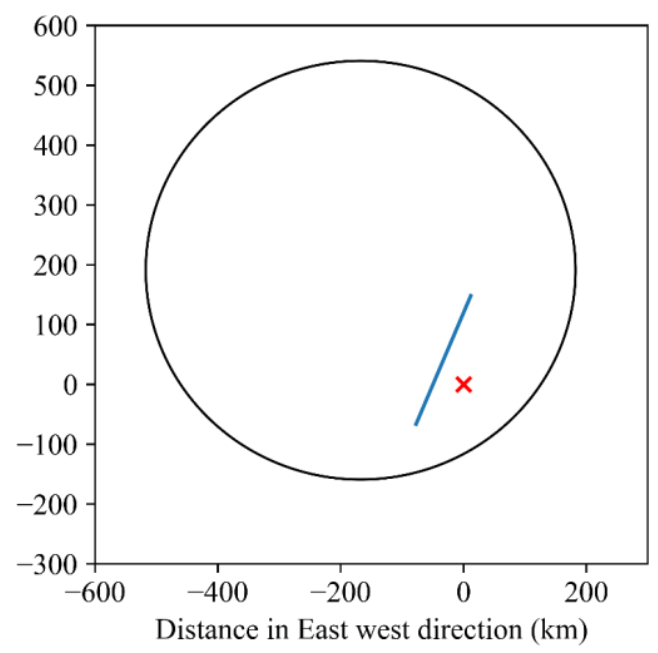

Figure 1. Geometry of the circular seismic zone (in black) and fault (in blue) relative to the site (in red) 
Table 1. Parameters used in ground motion simulations

\begin{tabular}{|ccc|}
\hline \multirow{2}{*}{ Source parameters } & $\beta$ & $3.6 \mathrm{~km} / \mathrm{s}$ \\
& $\Delta \sigma$ & $150 \mathrm{bar}$ \\
\hline \multirow{3}{*}{ Attenuation parameters } & $R_{1}, R_{2}$ & $70,130 \mathrm{~km}$ \\
& $p_{1}, p_{2}$ & $0,0.5$ \\
& $Q$ & $500 \mathrm{freq}^{0.65}$ \\
\hline Site parameters & $\kappa_{0}$ & 0.01 \\
\hline
\end{tabular}

catalog, one GM is simulated considering source to site distance and site characteristics using a stochastic simulation method (Boore 2003). The input parameters for the simulations are presented in Table 1 (Frankel et al 1996). Rather than using the median Fourier amplitude to simulate ground motions, each ground motion is obtained from a realization from the entire distribution of Fourier amplitude spectrum with standard deviation and correlation matrix borrowed from Bayless and Abrahamson (2019). The correlations between Fourier amplitudes and duration are estimated using a duration model developed by Bahrampouri et al. (2020) and a preliminary Fourier amplitude spectrum model developed based on the KiK-net database.

\section{CASE STUDY STRUCTURAL MODEL}

A 4-story concrete office building located in Charleston, SC is used to compare the impact of hazard scenario selection. The concrete building geometry and layout was selected to represent the typical concrete office construction in the Eastern US. As shown in Figure 2, the building consists of perimeter special-resisting moment frames in both directions, where typical span length and story height are taken as $30 \mathrm{ft}(9.14 \mathrm{~m})$ and $12 \mathrm{ft}(3.66 \mathrm{~m})$. Both lateral-resisting and gravity frames are constructed using concrete with a compressive strength of $5 \mathrm{ksi}(34.47 \mathrm{MPa})$ and A706 Gr.60 steel reinforcement. The framed floor structure is a 5" one-way slab concrete. The building is designed
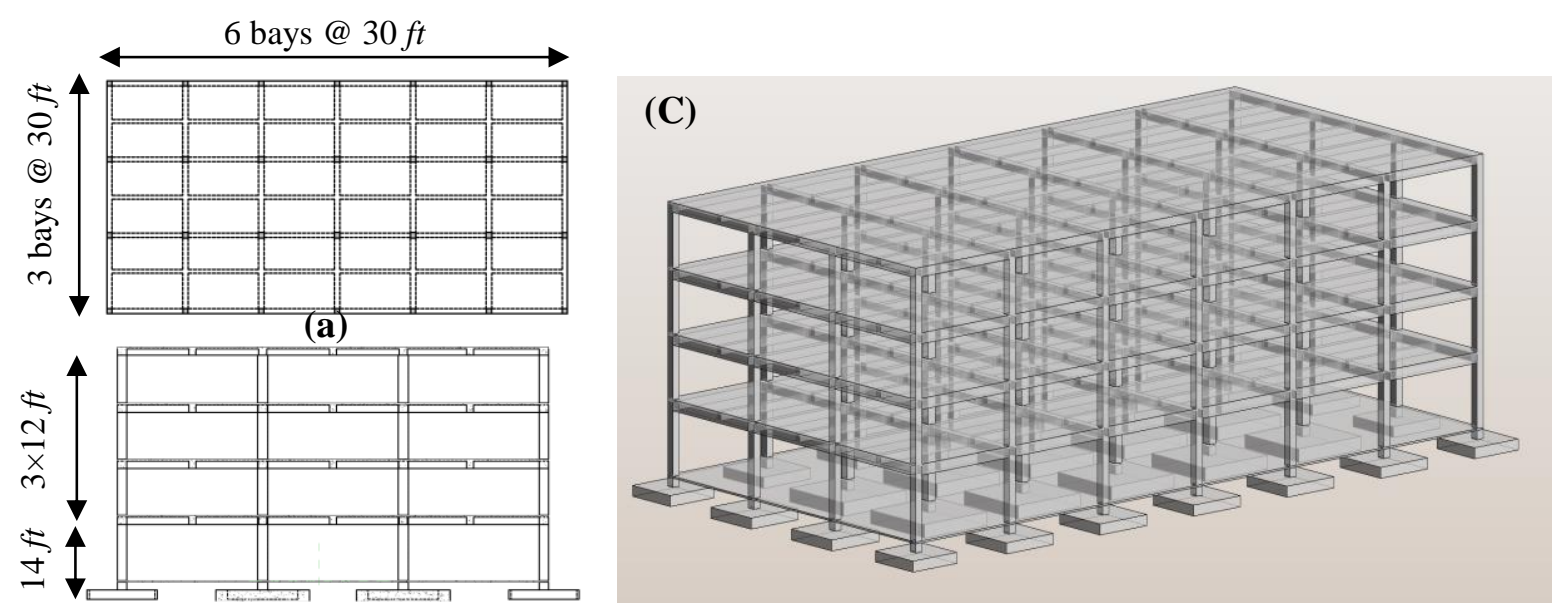

(b)

Figure 2. Archetype office building: (a) plan (b) elevation and (c) 3D view. 
for a site with stiff soil corresponding to mapped earthquake spectral acceleration at short periods $\left(\mathrm{S}_{\mathrm{s}}\right)$ and 1s $\left(\mathrm{S}_{1}\right)$ of $1.096 \mathrm{~g}$ and $0.348 \mathrm{~g}$, respectively. Using the ASCE 7-10 design spectra, this corresponds to a $S_{a}$ of $0.25 \mathrm{~g}$ for the structures first-mode period $\left(T_{l}=1.56 \mathrm{~s}\right.$ ). For the assumed seismicity of the site (Figure 1), the return period of this spectral acceleration is 2475 years.

A two-dimensional finite element model of the building is developed in OpenSees (McKenna 2011); Figure 3 shows the schematics of the developed nonlinear model. The frame elements are modeled using the concentrated plasticity model, where plastic hinges are located at both ends of the element. The hysteretic behavior of plastic hinges is then defined based on the peak-oriented Ibarra-Medina-Krawinkler (IMK) model (Ibarra et al. 2005). The equations proposed by Haselton et al. (Haselton et al. 2008) are used to derive the backbone curve of the IMK model in terms of effective stiffness $\left(K_{\text {eff }}\right)$, yielding moment $\left(M_{y}\right)$, pre-capping rotation $\left(\theta_{p}\right)$ and post-capping rotation capacity $\left(\theta_{p c}\right)$, and cyclic deterioration $(\lambda)$ of concrete members. Degradation of both stiffness and strength is accounted for in the numerical model. Steel02 and Concrete01 materials are used to define the constitutive behavior of reinforcing steel and concrete, respectively. The Method proposed by Zareian and Medina (2010) is used to adjust plastic hinges stiffness to resolve damping and convergence issues of the plastic hinges model. The P- $\Delta$ effect of interior gravity frames is considered using a fictitious leaning column that is connected to the lateral system using truss elements.

\section{HAZARD SCENARIO SELECTION}

To investigate the impact of hazard scenario selection, eight discrete hazard levels are selected which corresponds to exceedance probability of $1 \%$ in 200, $2 \%$ in 200 years, $2 \%$ in 100 years, $2 \%$ in 50 years, $5 \%$, in 75 years $10 \%$ in 75 years, $10 \%$ in 50 years and $10 \%$ in 21 years. These exceedance probabilities correspond to return periods of 19900, 9900, 2475, 1462, 712, 475, 199 years, respectively. For each hazard level, a uniform hazard spectrum is constructed and the $S_{a}$

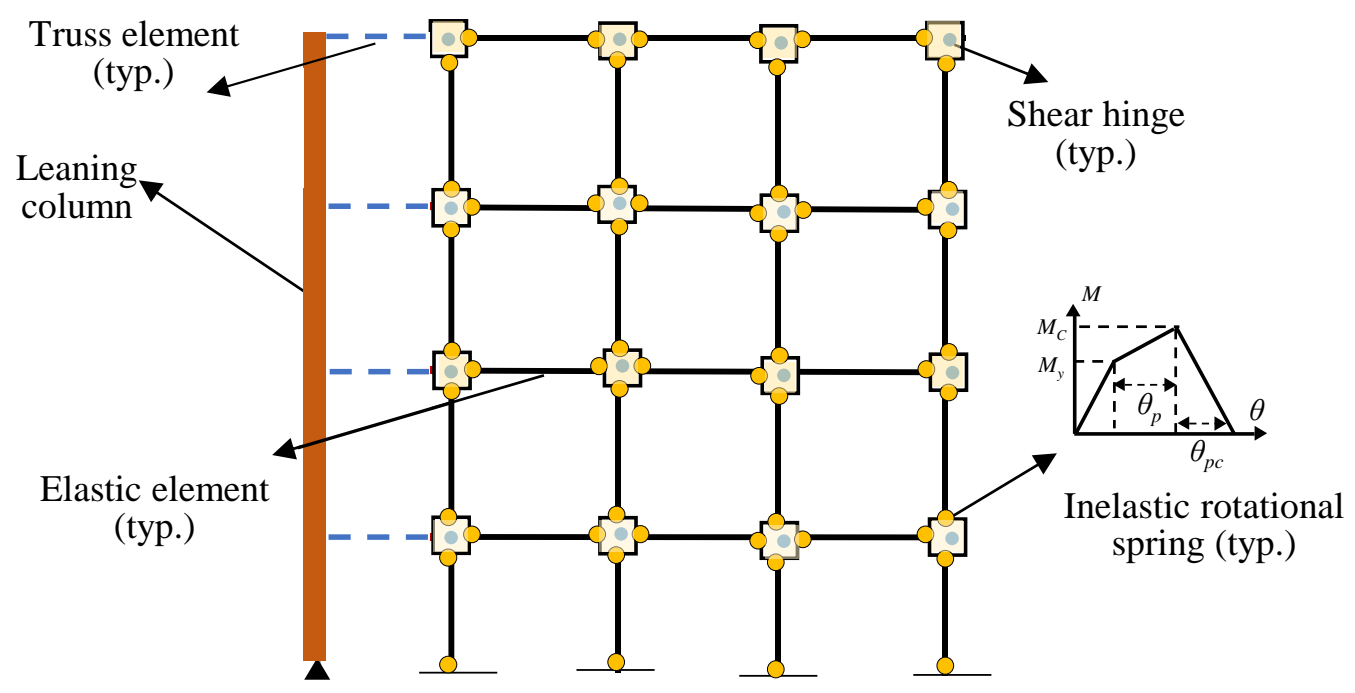

Figure 3. Nonlinear model configuration 
value at the structure's fundamental period $(\mathrm{T}=1.5 \mathrm{~s})$ is extracted to perform hazard deaggregation. Two hazard disaggregation sets are performed as follows (Fox et al. 2016):

$P\left(M, R \mid S_{a, L}<S_{a}<S_{a, U}\right)=\frac{\lambda\left(S_{a, L}<S_{a}<S_{a, U} \mid M, R\right)}{\lambda\left(S_{a, L}<S_{a}<S_{a, U}\right)}$

where $\lambda(x)$ denotes the mean annual rate of occurrence of $x$, and $S_{a, L}$ and $S_{a, U}$ shows the lower and upper bound of the $S_{a}$ range. The first hazard deaggregation formulation (referred to as IM occurrence) uses $0.9 S_{a}$ and $1.1 S_{a}$ as lower and upper bounds of any given $S_{a}$ value from the UHS, respectively. The second formulation (referred to as IM exceedance) mimic the current practice and uses $S_{a}$ value from the UHS value to $\infty$ as the lower and upper bound of Equation 1.
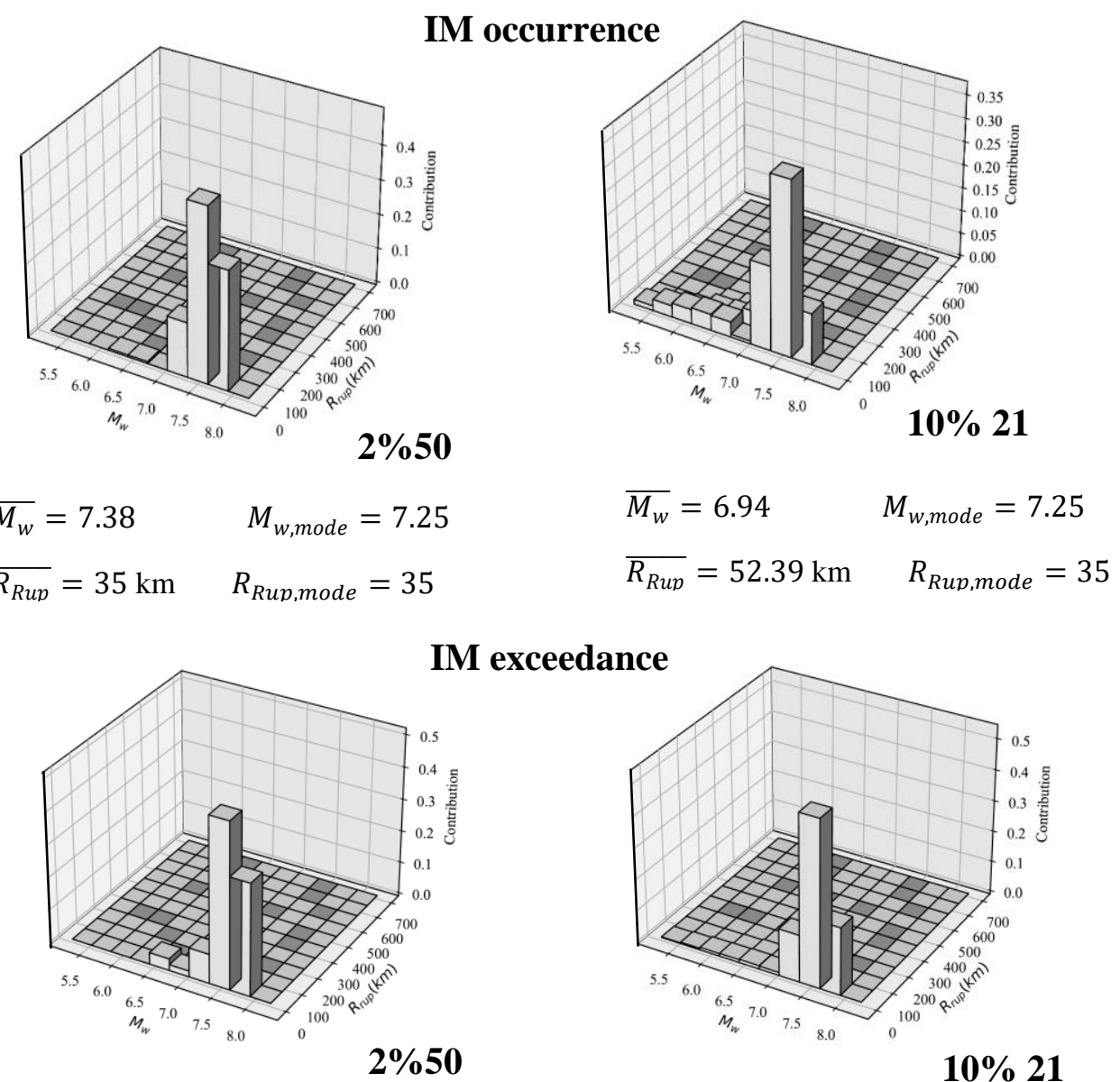

$\begin{array}{ll}\overline{M_{w}}=7.31 & M_{w, \text { mode }}=7.25 \\ \overline{R_{\text {Run }}}=35 \mathrm{~km} & R_{\text {Run.mode }}=35 \mathrm{~km}\end{array}$

$\overline{M_{w}}=7.19$

$10 \% 21$

Figure 4. Hazard deaggregation results for $S_{a}(T=1.5 \mathrm{~s})$ using different hazard deaggregation formulation for $2 \%$ in 50 years and $10 \%$ in 21 years 
Figure 4 shows the hazard deaggregation for two different hazard levels corresponding to $2 \%$ in 50 years (i.e. annual exceedance rate of $4.04 \times 10^{-4}$ ) and $10 \%$ in 21 years (i.e. annual exceedance rate of $\left.5.02 \times 10^{-3}\right)$. As shown in Figure 4, while the most contributing $M$ and $R$ scenario remains the same (same modal values), the average scenario changes based on the hazard formulation and selected hazard level.

\section{SEISMIC RISK ESTIMATION}

To estimate structural risk, nonlinear time history analyses are performed for the total ground motion suite (99,917 GM records) using computational resources at Virginia Tech's Advanced Research Computing (ARC) center. The building's response is recorded in terms of maximum inter-story drift (MIDR) and post-processed to obtain the fragility curve, which is the probability of exceeding a certain response threshold conditioned on the input GM's IM. While different methods exist to derive fragility curves (Baker 2015; Zaker Esteghamati and Huang 2019), a logistic regression formulation is adopted here to determine the probability that a given $S_{a}$ value results in building exceeding MIDR threshold as follows:

$$
P\left(M I D R>\operatorname{midr} \mid S_{a}\right)=\frac{1}{1+e^{-\left(a_{0}+a_{0} S_{a}\right)}}
$$

where $a_{0}$ and $a_{1}$ are logistic regression coefficients. For each selected set, seismic demand hazard curves are then calculated as follows:

$$
\lambda(M I D R>\text { midr })=\int_{S_{a}} P\left(M I D R>\operatorname{mid} r \mid S_{a}\right)\left|\frac{d \lambda\left(S_{a}\right)}{d S_{a}}\right| d S_{a}
$$

where $\lambda\left(S_{a}\right)$ shows the mean annual frequency of $S_{a}$ exceedance or IM hazard curve.

The benchmark seismic demand curve is obtained by adopting a range of MIDR values uniformly spaced from $0.3 \%$ to $5 \%$, and fitting a logistic regression to each MIDR level to estimate the conditional probability of exceeding the given MIDR threshold. The resulting fragility is then multiplied by the $S_{a}$ hazard curve and numerical integration is performed to derive the mean annual frequency of exceeding the given MIDR threshold.

Figure 5 shows the comparison of seismic demand hazard curves obtained from the four selected sets and the whole ground motion suite (referred to as "Benchmark"). In addition, slight, moderate, extensive, and complete damage states are shown based on HAZUS (Hazus 2012) recommendation for mid-rise concrete frame buildings. Using IM occurrence formulation both GM selection methods agree well for mid-range MIDR levels, where GMs selected based on average scenarios are closer to the benchmark (Figure 5a). Both methods show significant bias at small MIDRs, possibly due to neglecting small earthquake scenarios. Therefore both method overestimates annual exceedance of exceeding moderate damage, which is an important concern since moderate damage level dominates economic loss assessment of buildings. On the other hand, 


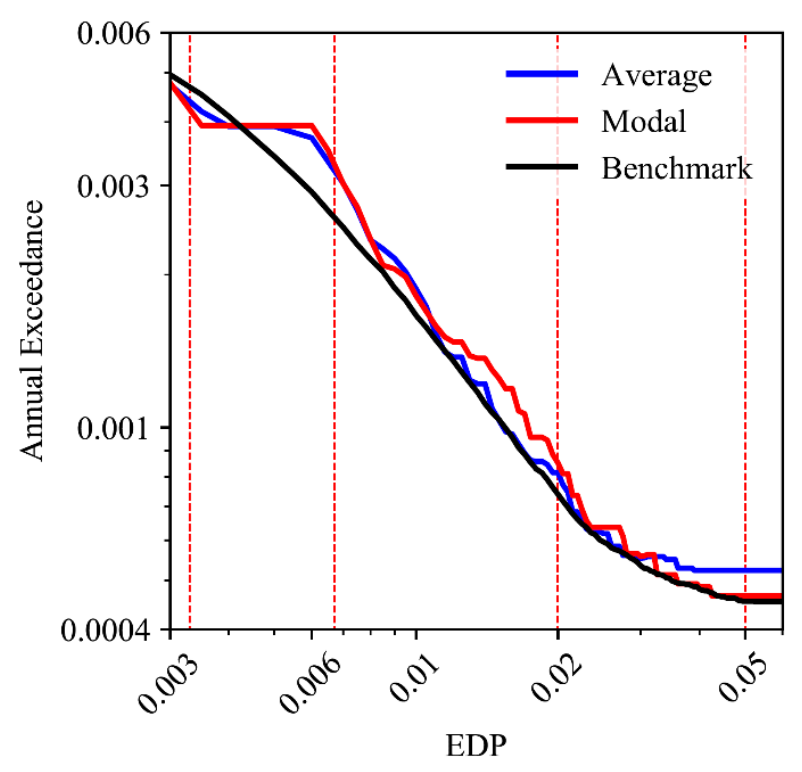

(a) IM occurrence

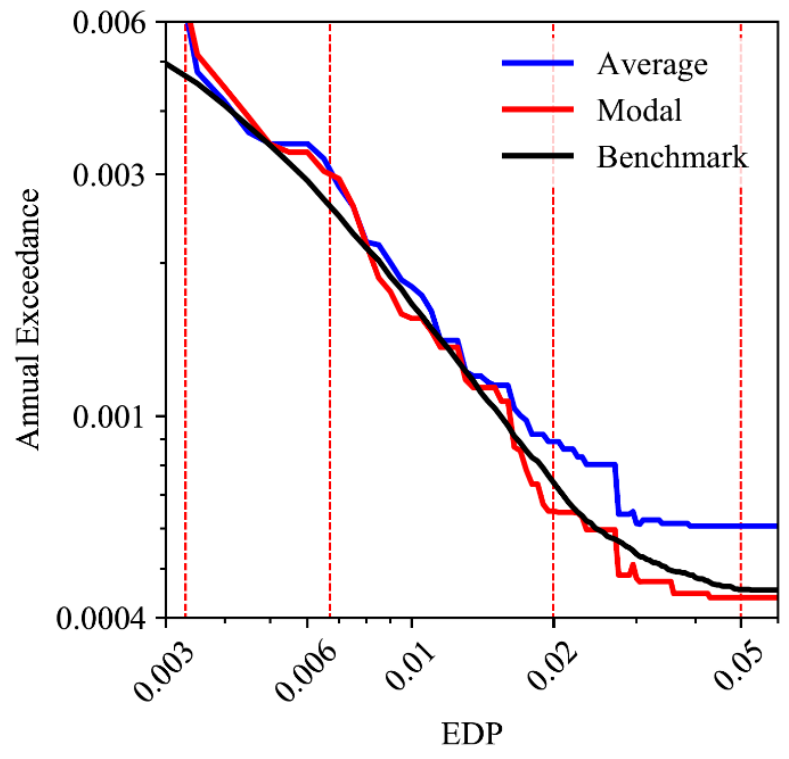

(b) IM exceedance

Figure 5. Comparison of demand curves obtained from modal and average scenarios to the benchmark demand curve for hazard deaggregation formulated based on (a) IM occurrence, and (b) IM exceedance

at the complete damage state, GMs selected to match modal scenarios are in close agreement with the benchmark. A possible explanation is that modal scenarios for this hypothetical site correspond only to stronger GMs that contribute to larger MIDR responses. Figure 5b shows the comparison between modal and average scenarios for the common hazard deaggregation formulation in terms of IM exceedance. As shown in Figure 5b, GMs selected to match modal scenarios are in good agreement with the benchmark, whereas average scenario-based GMs significantly deviates from the benchmark at the complete damage state. It should also be noted that the small MIDR bias from the two approaches is smaller in this case.

\section{CONCLUSION}

This study presents preliminary results on the impact of hazard deaggregation formulation and scenarios on structural risk estimation. A Monte Carlo seismic analysis was performed and combined with a stochastic GM simulation module to represent a hypothetical site's seismic activity during a time span of 1,000,000 years. Subsequently, four ground motion sets are selected from the derived benchmark, each matching to one statistic of the site's hazard deaggregation (mean and mode) and the hazard deaggregation formulation (IM exceedance or occurrence). The results demonstrate that using IM occurrence formulation, while GMs selected to match the average scenario can better represent the structural risk at mid-range damage levels, they deviate from the benchmark at higher damage states. On the other hand, GM selected to match the modal 
scenario better capture MIDR risk at higher damage states, while deviates from the benchmark elsewhere. For IM exceedance formulation, mode-based GMs overall better match the benchmark. Therefore, the results suggest that there is an interaction between hazard formulation and scenario statistics; and neither average nor modal scenarios can precisely capture expected seismic risk at

the full spectrum of structural response, even for a site with unimodal deaggregation, warranting further research on a more refined approximation of hazard deaggregation.

\section{ACKNOWLEDGMENT}

The authors wish to acknowledge the help provided by Advanced Research Computing (ARC) at Virginia Tech, particularly guidance provided by Matthew Brown on parallel computation, that facilitated the large number of time history analyses performed in this study.

\section{REFERENCES}

Bahrampouri, M., Rodriguez-Marek, A., and Green, R. A. (2020). "Ground Motion Prediction Equations for Significant Duration Using the Kik-Net Database.” Earthquake Spectra.

Baker, J. W. (2011). "Conditional Mean Spectrum: Tool for Ground-Motion Selection.” Journal of Structural Engineering.

Baker, J. W. (2015). "Efficient analytical fragility function fitting using dynamic structural analysis." Earthquake Spectra.

Bayless, J., and Abrahamson, N. A. (2019). "An empirical model for the interfrequency correlation of epsilon for fourier amplitude spectra." Bulletin of the Seismological Society of America.

Boore, D. M. (2003). "Simulation of ground motion using the stochastic method." Pure and Applied Geophysics, 160(3-4), 635-676.

Bradley, B. A. (2012). "A ground motion selection algorithm based on the generalized conditional intensity measure approach." Soil Dynamics and Earthquake Engineering.

Fox, M. J., Stafford, P. J., and Sullivan, T. J. (2016). "Seismic hazard disaggregation in performance-based earthquake engineering: occurrence or exceedance?” Earthquake Engineering \& Structural Dynamics, Wiley Online Library, 45(5), 835-842.

Frankel, A., C. Mueller, T. Barnhard, D. Perkins, E. Leyendecker, N. Dickman, S. Hanson, and M. Hopper (1996). National seismic hazard maps, June 1996, U.S. Geol. Surv. Open-File Rept. 96-532.

Haselton, C. B., Liel, A. B., and Lange, S. T. (2008). "Beam-Column Element Model Calibrated for Predicting Flexural Response Leading to Global Collapse of RC Frame Buildings." Peer 2007, 03(May).

Hazus. (2012). Hazus-MH 2.1: Technical Manual. Federal Emergency Management Agency.

Ibarra, L. F., Medina, R. A., and Krawinkler, H. (2005). "Hysteretic models that incorporate strength and stiffness deterioration." Earthquake Engineering and Structural Dynamics, 34(12), 1489-1511.

Iervolino, I., Chioccarelli, E., and Convertito, V. (2011). "Engineering design earthquakes from multimodal hazard disaggregation." Soil Dynamics and Earthquake Engineering.

Kohrangi, M., Vamvatsikos, D., and Bazzurro, P. (2020). "Multi-level conditional spectrumbased record selection for IDA.” Earthquake Spectra. 
Kwong, N. S., and Chopra, A. K. (2016). "Evaluation of the exact conditional spectrum and generalized conditional intensity measure methods for ground motion selection."

Earthquake Engineering and Structural Dynamics, 45(5), 757-777.

Kwong, N. S., and Chopra, A. K. (2020). "Selecting, scaling, and orienting three components of ground motions for intensity-based assessments at far-field sites." Earthquake Spectra, SAGE Publications Sage UK: London, England, 8755293019899954.

Lin, T., Harmsen, S. C., Baker, J. W., and Luco, N. (2013a). "Conditional spectrum computation incorporating multiple causal earthquakes and ground-motion prediction models." Bulletin of the Seismological Society of America.

Lin, T., Haselton, C. B., and Baker, J. W. (2013b). "Conditional spectrum-based ground motion selection. Part I: Hazard consistency for risk-based assessments.” Earthquake Engineering and Structural Dynamics.

McKenna, F. (2011). "OpenSees: A framework for earthquake engineering simulation." Computing in Science and Engineering.

Musson, R. M. W. (2000). "The use of Monte Carlo simulations for seismic hazard assessment in the U.K." Annali di Geofisica.

Simon Kwong, N., and Chopra, A. K. (2017). "A Generalized Conditional Mean Spectrum and Its Application for Intensity-Based Assessments of Seismic Demands." Earthquake Spectra.

Talwani, P., and Chapman, M. C. (2006). Seismic Hazard Mapping for Bridge and Highway Design in South Carolina.

Tarbali, K., and Bradley, B. A. (2015). "Ground motion selection for scenario ruptures using the generalised conditional intensity measure (GCIM) method." Earthquake Engineering and Structural Dynamics.

Zaker Esteghamati, M., and Huang, Q. (2019). "An efficient stratified-based ground motion selection for cloud analysis. " 13th International Conference on Applications of Statistics and Probability in Civil Engineering (ICASP13), Seoul, South Korea.

Zaker Esteghamati, M., Lee, J., Musetich, M., and Flint, M. M. (2020). "INSSEPT: An opensource relational database of seismic performance estimation to aid with early design of buildings." Earthquake Spectra, doi:10.1177/8755293020919857.

Zareian, F., and Medina, R. A. (2010). "A practical method for proper modeling of structural damping in inelastic plane structural systems." Computers and Structures, 88(1-2), 45-53. 